\title{
A QUDA-branch to compute disconnected diagrams in GPUs
}

\section{Constantia Alexandrou}

Department of Physics, University of Cyprus, P.O. Box 20537, 1678 Nicosia, Cyprus, and Computation-based Science and Technology Research Center, Cyprus Institute, 20 Kavafi Str., Nicosia 2121, Cyprus

E-mail: alexandeucy.ac.cy

\section{Kyriacos Hadjiyiannakou}

Department of Physics, University of Cyprus, P.O. Box 20537, 1678 Nicosia, Cyprus E-mail: hadjigiannakou.kyriacos@ucy.ac.cy

\section{Giannis Koutsou}

Computation-based Science and Technology Research Center, Cyprus Institute, 20 Kavafi Str., Nicosia 2121, Cyprus

E-mail: g.koutsoudcyi.ac.cy

\section{Alexei Strelchenko}

Scientific Computing Division, Fermilab, Batavia, IL 60510-5011, USA

E-mail: astrelefnal.gov

\section{Alejandro Vaquero Avilés-Casco*}

Computation-based Science and Technology Research Center, Cyprus Institute, 20 Kavafi Str., Nicosia 2121, Cyprus

E-mail: a.vaquero@cyi.ac.cy

Although QUDA allows for an efficient computation of many QCD quantities, it is surprinsingly lacking tools to evaluate disconnected diagrams, for which GPUs are specially well suited. We aim to fill this gap by creating our own branch of QUDA, which includes new kernels and functions required to calculate fermion loops using several methods and fermionic regularizations.

31st International Symposium on Lattice Field Theory - LATTICE 2013

July 29 - August 3, 2013

Mainz, Germany

\footnotetext{
*Speaker.
} 


\section{Introduction}

Graphics Processing Units (GPUs) are swiftly changing the panorama of lattice QCD, due to the unprecedented increase of computer power provided by the graphic cards. Nonetheless, lack of efficient libraries for specific task usually prevents scientist from benefiting from the raw processing power the GPUs offer. A great effort has been done in this direction with the nVidia-based QUDA library $[1,2]$, featuring many tools to generate and analyze configurations.

Although the QUDA library is fairly complete at the present moment, it is still under development, and new capabilities are added every day. One of the capabilities we were missing was specific code for calculating disconnected diagrams; the present work is devoted to fill this empty space.

\section{Integrating the different methods in QUDA}

In order to calculate disconnected diagrams, we mainly need to solve the equation

$$
M\left|s_{r}\right\rangle=\left|\eta_{r}\right\rangle,
$$

but QUDA already has a large set of inverters. The previous step of generating the random source is done at this moment in CPUs, using external libraries for high quality random number generation (although inclusion in GPUs is planned in the near future). In this section we will mostly deal with the specific variation reduction techniques, adapted for GPU computation.

\subsection{Implementation of the Truncated Solver Method}

The first variation reduction technique we implemented on GPUs is the Truncated Solver Method (TSM) [3]. In fact, the TSM was our main motivation to move from CPUs to GPUs our calculations of disconnected diagrams. The reason behind this moving is the high efficiency of the GPUs when dealing with low precision numbers: in current GPU architectures, computer power is bound to memory bandwidth. Therefore a reduction in the precision (and consequently in the size of the data read/written to/from memory) results in an immediate increase of the flop count. In fig. 1 it is shown how the computer power roughly halves as the size of numerical data is duplicated, from half to single, and from single to double.

The TSM relies on a low precision estimation of the inverse of the fermionic matrix with high statistics, corrected by a high precision computation at low statistics. For the low precision estimation we use around $\sim 10^{2}-10^{3}$ inverted at low precision $\left(\rho \sim 10^{-2}\right)$, for which half precision numbers are suitable. The correction, in contrast, requires much higher precision $\left(\rho \sim 10^{-8}\right)$, and half precision solvers will not converge to desired precision. We need at least a single precision solver, which, as shown in fig. 1, is not as efficient as the half precision solver. Nonetheless, we only need to perform a few $(\sim 10-100)$ high precision inversions to get a good correction in most cases, therefore most of the time is spent in the low precision calculation, which is the one with improved speed. 

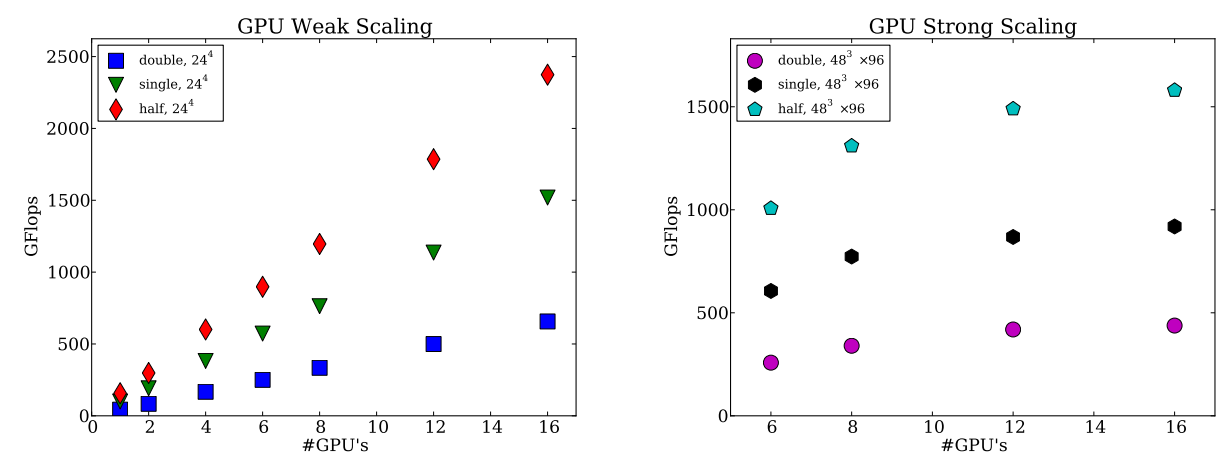

Figure 1: GPU scaling performance of the twisted mass CG solver of the QUDA library in Tesla m2070 cards. Each node of the cluster featured two GPUs, and the nodes were interconnected with infiniband.

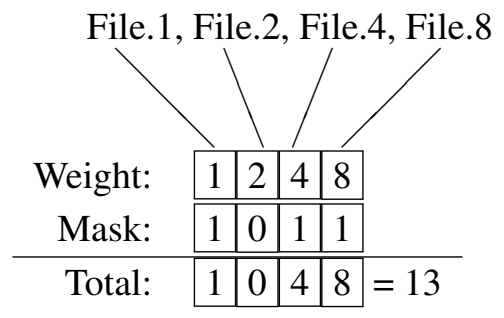

Figure 2: Example of construction of the inverse estimator using 13 sources in our storage method.

\subsubsection{The problem of storage}

When the TSM is introduced, one has to face the problem of storage and take decisions regarding which information can be discarded, due to the large number of stochastic sources generated. In our case it was decided that only contractions would be stored, but even in this case a storage problem might appear.

For our calculations, we mainly used the one-end trick for twisted mass fermions (recently included in QUDA [4]), which requires volume sources and gives results for all time-slices. We computed all ultra-local and one-derivative insertions as well, with and without a flavor $\tau_{3}$ matrix, that is, 160 insertions in total. We also calculated the data for several values of the momenta, up to $p^{2}<=9$. In the end, each configuration with volume $32^{3} \times 64$ was taking $\sim 50 \mathrm{~Gb}$ storage, a monster number taking into account that GPU application usually are not granted as much disk storage as CPU's. In the GPU clusters we run our code, we were granted between 5 and $20 \mathrm{~Tb}$ of disk space, which we would fill with 100-400 configurations, a number that might hardly be enough for a single ensemble, let alone when dealing with several ensembles.

An obvious way to reduce storage needs is to transform the data from text to binary format, which will grant us a $\sim 70 \%$ reduction in disk usage, although it will be still a huge amount of data. A further reduction of storage requirements is only achieved through clever techniques; in our case we developed a binary-storage technique, inspired in the way the bits make up a byte. This technique reduced storage requirements up to $\sim 97 \%$ without losing any relevant information.

In order to understand the way the technique works, we can have a look at fig. 2 . The byte 
is composed of bits, and each bit has a different wait according to its position $(1,2,4 \ldots)$. The idea is to mimic this structure for the stochastic sources. Since in the end we are going to average the sources, we can add several and store the addition in a single file; so in File.1 file we store the contractions generated with one stochastic source, in File. 2 we store the sum of the contractions coming from the second and the third stochastic source, and so on. Reconstruction is straightforward, taking into account the base-2 structure.

With this storage method one can recover the data for any number of sources, therefore we are keeping the same information in much less space. Actually some information is lost, for after storing the data there is only one way to recover a fixed number of sources, whereas before there could be many, but this extra information is not useful for us and can be discarded. In contrast, we gain a huge reduction in storage requirements, from $O(N)$ to $O\left(\log _{2} N\right)$.

\subsection{Contraction kernels}

A fundamental step in the calculation of loop amplitudes is the contraction of inverted sources. To this end we developed efficient GPU code, for the contraction step can be parallelized very easily. As a result, our contractions are done at speeds of $\sim 300$ GFlops in a single Tesla m2070 GPU in double precision, and the contraction code shows almost perfect scaling with increasing nodes.

Traces were taken in color space, leaving the Dirac and volume indices open. The volume indices are used later for the FFT, so we obtain solutions for different momenta, whereas the open Dirac indices are there in order to deal with the different insertions. We calculated the outer product in Dirac space of both sources to be contracted, and consequently a $4 \times 4$ matrix was obtained, with enough information to reconstruct any arbitrary $\gamma$ insertion just by transposition and multiplication. Therefore, our contraction code automatically outputs all the possible insertions for ultra-local operators. A covariant-derivative kernel is included, which gives correct results, but in our tests it was revealed that the contraction performance was not so good when including one-derivative insertions.

In our implementation, we mainly worked with twisted mass fermions and the one-end trick [5], but we also worked with time-dilution [6]. Therefore we developed GPU kernels to contract a single time slice or a whole vector, returning an array with a time-slice index.

\subsection{Interfaces}

Since QUDA already implements most of the code we need for computing disconnected diagrams, the largest contribution to the library on this package is the writting of interfaces. Those were designed to calculate any ultra-local and one-derivative insertion with several variance reduction methods, namely the TSM, the one-end trick (only for twisted mass fermions), time-dilution and the Hopping Parameter Expansion (HPE) [7], and all the possible combinations of these.

The interface generates random stochastic sources using RANLUX from the GSL library on the CPUs; then the source is sent to the GPUs for inversion and contraction, and contractions are stored back in the CPUs. This process is repeated several times for the binary storage system. After we accumulated enough sources, the data is sent back from CPUs to GPUs for FFT using the nVidia library cuFFT; our output from the last section fits exactly in the input required in the 


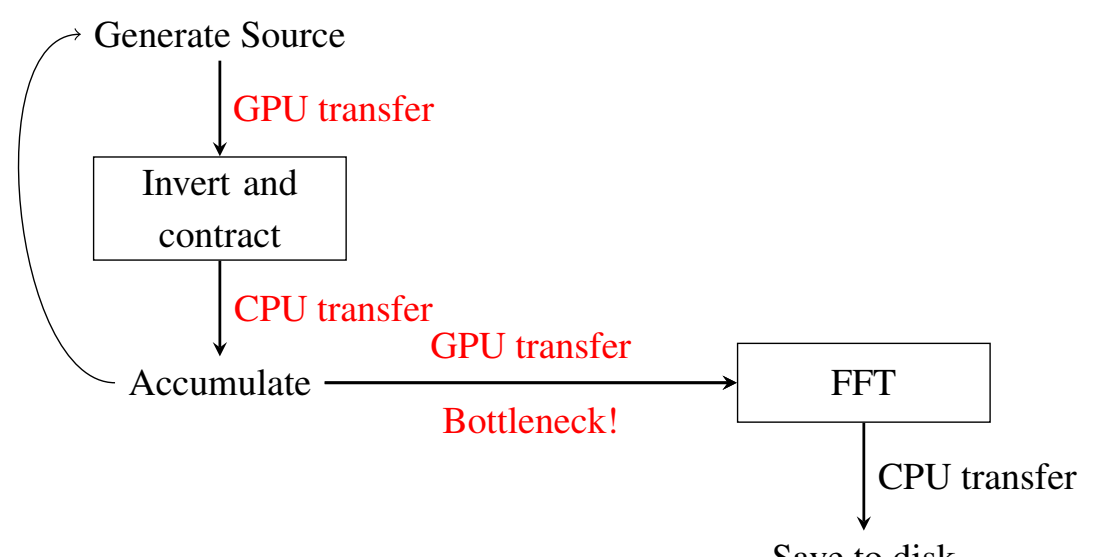

Save to disk

Figure 3: Implemented scheme in the current version of the discLoop package. The job runs in CPUs, unless the computation step is inside a box, in that case it runs in GPUs. In red, the detected bottlenecks.

functions of the cuFFT library, so no further transformations are required. At this point, all possible momenta are generated, but we usually insert here a cut-off in $p^{2}$ to reduce storage. Finally, the results are written to disk in a parallel fashion to reduce $\mathrm{I} / \mathrm{O}$ time.

\subsection{Limitations and bottlenecks}

Although in our implementation the tough numerical work is done by the GPUs, many operations are still performed in CPUs, so there is a lot of room for improvement. Memory transfers between CPU and GPU are frequent, and although these are never done in critical spots of the code, we can yet see performance gains if we move more tasks to the GPUs. Fig. 3 gathers all these problems in a simpel diagram.

The first bottleneck appears in the generation of the source, we could simply use cuRAND to get random sources directly on the GPUs. Further on, and after inversion and contraction, the step of accumulation of the sources introduces a serious overhead coming from memory transfers, mainly when dealing with low precision sources in the TSM, for the inversion of these sources can take in some scenarios a fraction of a second. Unfortunately, memory constraints might now allow us to accumulate in GPUs, this mainly depends on the number of operators being computed and the variance reduction technique used: the one-end trick for instance requires volume sources, and when it is combined with one- derivative insertions, the amount of data is simply to large to deal with. But other combinations can be easily solved, like the one-end trick with ultra-local operators or time-dilution with any kind of operator. Our ideal diagram would look like the one shown in fig. 4.

Apart from the bottlenecks, the current code suffers from several limitations:

- Splitting is only allowed in the time-direction, and although splitting in other directions can be implemented, it is not trivial and will require some time.

- MultiGPU is currently only supported through MPI, although an update in the code should fix this limitation. From the QUDA library a huge effort has been done in order to make the 


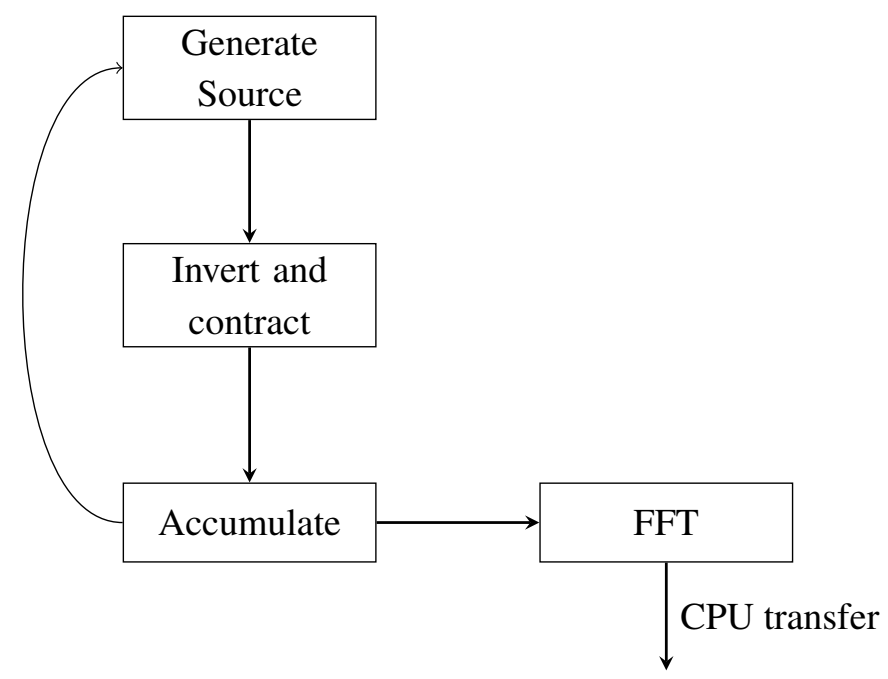

Save to disk

Figure 4: Projected scheme for the package, with most bottlenecks removed and all work done in GPUs.

communications layer more and more abstract, so these kind of limitations are removed.

- Only twisted-mass fermions are supported at this moment. Most likely the package will work with Wilson (and clover) fermions, but this has not been extensively tested.

In spite of these limitations, we are currently using the code in production phase very successfully.

\subsection{Future plans and conclusions}

The successful application of GPUs to the problem of disconnected diagrams allows for an unprecedented precision in this kind of calculations, due to the huge numerical power available. In our tests, the stochastic error coming from the fact that the loop amplitudes were being estimated was greatly reduced, or even dissappeared, rendering the disconnected diagrams accessible. The obstacle that prevented the application of GPUs to this particularly tough problem was the implementation of efficient code in GPUs, which always is a very difficult task. For that reason, it is very important to invest in the development of libraries (like QUDA) that can simplify tasks for scientist. Our package aims to become an easy-to-apply but efficient and powerful solution for computing disconnected diagrams.

The next plans for the package consist of a clean-up and removal of critical limitations, so it can be merged with master branch of QUDA as soon as possible. Once this feat is accomplished, we can focus in expanding the current implementation to include other variance reduction techniques, as color- and/or spin-dilution, or in optimizing the code to increase performance and reduce the bottlenecks. Nonetheless the code as-is can be safely used for production purposes. It is available at GitHub and it is maintained periodically.

\section{Acknowledgments}

Alejandro Vaquero is supported by funding received from the Cyprus RPF under contract 
EPYAN/0506/08. This research was in part supported by the Research Executive Agency of the EU under Grant Agreement number PITN-GA-2009-238353 (ITN STRONGnet) and the infrastructure project INFRA-2011-1.1.20 number 283286, and the Cyprus RPF under contracts KY-ГА/0310/02 and NEA YПO $\Delta \mathrm{OMH} / \Sigma T P A T H / 0308 / 31$. This work has been partly supported by the PRACE-1IP and PRACE-2IP (Community Codes Development - Work Package 8) projects funded by the EUs 7th Framework Programme (FP7/2007-2013) under grant agreement no. RI-211528 and no. RI283493 respectively, and by SciDAC 2 project. Computer resources were provided by Cy-Tera at CaSToRC, Forge at NCSA Illinois (USA) and Minotauro at BSC (Spain).

\section{References}

[1] M. A. Clark et al., Comput. Phys. Commun. 181 (2010), 1517, arXiv: 0911.3191.

[2] R. Babich et al.,SC 2011, arXiv:1109.2935.

[3] G. Bali, S. Collins and A. Schäffer, PoSLaT2007, 141, arXiv: 0709. 3217.

[4] A. Strelchenko, C. Alexandrou, G. Koutsou, A. Vaquero, PoSLaT2013, 415, arXiv:1311. 4462.

[5] M. S. Foster and C. Michael, Phys. Rev. D59 (1999), 074503, arXiv: hep-lat/9810021; C. McNeile and C. Michael, Phys. Rev. D73 (2006), 074506, arXiv: hep-lat / 0603007.

[6] S. Bernardson, P. McCarty and C. Thron, Comput. Phys. Commun. 78 (1993), 256.

[7] C. Michael, M. S. Foster and C. McNeile, Nucl. Phys. Proc. Suppl. 83 (2000), 185, arXiv:hep-lat/9909036. 